\title{
Composite poly(vinyl alcohol)/poly(vinyl acetate) electrospun nanofibrous mats as a novel wound dressing matrix for controlled release of drugs
}

This article was published in the following Dove Press journal:

International Journal of Nanomedicine

19 May 201 I

Number of times this article has been viewed

\author{
Marziyeh Jannesari' \\ Jaleh Varshosaz ${ }^{2}$ \\ Mohammad Morshed' \\ Maedeh Zamani' \\ 'Department of Textile Engineering, \\ Isfahan University of Technology, \\ Isfahan, Iran; ${ }^{2}$ Department of \\ Pharmaceutics, School of Pharmacy \\ and Pharmaceutical Sciences Research \\ Center, Isfahan University of Medical \\ Sciences, Isfahan, Iran
}

\begin{abstract}
The aim of this study was to develop novel biomedicated nanofiber electrospun mats for controlled drug release, especially drug release directly to an injury site to accelerate wound healing. Nanofibers of poly(vinyl alcohol) (PVA), poly(vinyl acetate) (PVAc), and a 50:50 composite blend, loaded with ciprofloxacin $\mathrm{HCl}(\mathrm{CipHCl})$, were successfully prepared by an electrospinning technique for the first time. The morphology and average diameter of the electrospun nanofibers were investigated by scanning electron microscopy. X-ray diffraction studies indicated an amorphous distribution of the drug inside the nanofiber blend. Introducing the drug into polymeric solutions significantly decreased solution viscosities as well as nanofiber diameter. In vitro drug release evaluations showed that both the kind of polymer and the amount of drug loaded greatly affected the degree of swelling, weight loss, and initial burst and rate of drug release. Blending PVA and PVAc exhibited a useful and convenient method for electrospinning in order to control the rate and period of drug release in wound healing applications. Also, the thickness of the blend nanofiber mats strongly influenced the initial release and rate of drug release.
\end{abstract}

Keywords: biodegradable polymers, drug delivery, controlled release, electrospun nanofibers, wound dressing

\section{Introduction}

A wound dressing should ideally provide an optimal healing environment in which healing can occur at a maximum rate compatible with the cosmetic appearance of the healed wound; ${ }^{1,2}$ however, the infection under dressing caused by burns, split skin graft donor sites, pressure, sores, and diabetic ulcers can lead to prolonged healing time or, in serious conditions, death. Consequently, wounds often require treatment with antibiotics. ${ }^{2-5}$ Local delivery of drugs sustained for about 1 week is preferred to systemic administration, especially for chronic wounds, ${ }^{5}$ because of the advantages of these delivery systems such as: a) improving the effectiveness of drug therapy, and selective targeting, $b$ ) decreasing the side effects on a tissue, and interference with wound healing, c) reduction of bacterial resistance, and d) reducing the frequency of dressing replacements to increase patient compliance. ${ }^{4,5}$ Furthermore, a proper wound dressing needs to have wound healing properties, including the essential swelling capability for absorbing excess exudates and oxygen permeability for respiring., ${ }^{5,6}$ Modern dressings as vehicles for delivering therapeutic agents to wound sites have been applied in the form of hydrogels, ${ }^{2}$ films, ${ }^{7}$ sponges, ${ }^{8}$ foams, ${ }^{9}$ and recently in the form of nanofibers. ${ }^{6}{ }^{10}$ However, little research has been published on the controlled delivery of drugs from polymeric dressing, especially in the form of nanofibers. 
In the last decade, nanofibers have attracted increasing attention for their use in biomedical applications such as wound dressings, ${ }^{10-12}$ tissue engineering, ${ }^{11}$ and controlled drug delivery. ${ }^{13-21}$ The very fine diameter and high porous structure of nanofiber mats help drug particles diffuse out of the matrix more efficiently. ${ }^{14,15}$

The most popular, preferred, and inexpensive technique for fabricating polymeric nanofibers is electrospinning. Unlike common techniques, electrospinning is more convenient for incorporating therapeutic compounds into the polymeric matrix. ${ }^{11}$ Using electrospun nanofiber mats for wound dressings also has some benefits, such as hemostatic properties, absorbability and semipermeability for respiration of cells, better conformation to the wound surface, ${ }^{11}$ and the potential for leaving no scar. ${ }^{11,12}$

Among wound dressings, special attention has been paid to biodegradable polymeric dressings because they can easily be washed off the wound surface. ${ }^{4,5}$ Poly(vinyl alcohol) (PVA) is a well-known, biologically friendly, biodegradable polymer owing to its desirable properties such as nontoxicity, no carcinogenicity, biocompatibility, and appropriate mechanical properties. ${ }^{1,2,7,18}$ Because of these properties, PVA is used in some biomedical applications such as soft contact lenses, implants of artificial organs, cartilage skin, and cardiovascular devices. ${ }^{2,18}$ Because of its flexibility and swelling capability in an aqueous medium, PVA has been much studied as a wound dressing; ${ }^{2}$ however, its poor stability in water has limited its use in aqueous systems, particularly for drug-delivery applications. ${ }^{18,22}$ To overcome this problem, PVA has been made insoluble by grafting, ${ }^{18}$ copolymerizing, ${ }^{22}$ and cross-linking, ${ }^{23}$ which require some additional and sometimes complicated and time-consuming processes. In wound dressing applications, PVA has been crosslinked with polyvinyl pyrrolidone, ${ }^{2}$ sodium alginate, ${ }^{24}$ and sterculia ${ }^{25}$ to provide better characteristics, although a vey quick release burst was reported and a sustained release continued up to 90 hours, 5 hours, and 24 hours, respectively. Poly(vinyl acetate) (PVAc), a biocompatible and biodegradable (owing to the hydrolyzable groups in the side chain) polymer, has also been used in biomedical applications, including drug and cell carriers, and tissue engineering. ${ }^{26,27}$

PVA is a well-known biocompatible and nontoxic polymer. Biocompatibility of PVA is due to both its compatibility with tissue and blood (plasma proteins), which has been widely reported. For this reason, PVA has been used in numerous devices in contact with blood and tissues such as drug-delivery systems, wound dressings, dialysis membranes, cardiovascular devices, artificial cartilage, tendon, ligament, cornea, lens, skin, and intervertebral disc. ${ }^{4,23,28}$ A good blood-contact property was observed for pure PVA. ${ }^{29}$ Moreover, bovine serum albumin (BSA) tends to adsorb to hydrophobic surfaces more than hydrophilic ones. ${ }^{30}$ PVA has good blood compatibility. ${ }^{24}$ Ex vivo experiments with canine whole blood have shown that formation of thrombus on PVA is less than on siliconized glass. ${ }^{31}$ Methods such as blending, grafting, and copolymerization of PVA with other polymers have improved blood compatibility of those polymers, indicating a very high blood compatibility of PVA.

Several reports have indicated blood compatibility of PVAc. PVAc has also been applied in many medical fields because of its biocompatibility. Hydrogels such as PVAc containing functional groups such as $\mathrm{COOH}$ usually show good biocompatibility in contact with blood, body fluids, and tissues. ${ }^{32}$ In another study, PVAc did not adsorb serotonin from platelet-free plasma, and did not cause lysis of erythrocytes. ${ }^{33}$ PVAc is an inert polymer with the advantage that it does not induce a deleterious reaction in living tissue. Histological study of the embolized rat kidney revealed no detectable damage in the vessel wall and no recanalization for up to 6 months. Also it was shown that PVAc remained inert in blood vessels. ${ }^{34}$ Several patents are registered for novel compositions suitable for use in embolizing blood vessels using biocompatible polymers including PVAc. The composition of these inventions are particularly useful in embolizing blood vessel $\mathrm{s}^{35}$ or they can be applied to intravascular medical devices. ${ }^{36}$ The amount of serum albumin adsorbed onto the PVAc latex is considerably low, especially at a $\mathrm{pH}$ of 5 to 8 , indicating its good compatibility with blood. ${ }^{37}$ There were no procedural complications, and no thrombus formation on the electrodes of the PVAc catheter. ${ }^{38}$ Several studies have shown that PVAc improves blood compatibility of some polymers, thus demonstrating the good blood compatibility of this polymer. ${ }^{39}$

Ciprofloxacin, a fluoroquinolone antibiotic, is one of the most widely used antibiotics in wound healing because of its low minimal inhibitory concentration for both Gram-positive Staphylococcus and Gram-negative Pseudomonas bacteria that cause wound infections. ${ }^{2,3}$

In the present research, an economical and convenient method of blending was applied to achieve a simultaneous controlled release of $\mathrm{CipHCl}$ and high swelling capacity along with coverage conformability for wound healing administration. PVAc, as an inexpensive and hydrophobic polymer, was selected to be blended with PVA solution. 
While PVAc is insoluble in water (a good solvent of PVA), ${ }^{18}$ an aqueous solution of $50 \%$ acetic acid was used as an appropriate solvent to dissolve both polymers and also $\mathrm{CipHCl}$ to attain a stable blend solution. The resulting solution was electrospun into nanofibers.

\section{Materials and methods Materials}

PVA (MW =72,000 Da, hydrolysis 98\%) and acetic acid were purchased from Merck Chemical Co (Darmstadt, Germany), PVAc (MW = 55,000-70,000 Da) was obtained from Carl Roth $\mathrm{GmbH}$ (Karlsruhe, Germany), and $\mathrm{CipHCl}$ was supplied from TEMAD Co (Tehran, Iran).

\section{Preparation of pure and drug-loaded nanofiber mats}

Aqueous acetic acid solution diluted to $50 \%$ with distilled water was used as the solvent for PVA and PVAc. The PVA solutions were prepared by dissolving $5 \% \mathrm{w} / \mathrm{v}$ of PVA powder in $50 \%$ acetic acid solution at $70^{\circ} \mathrm{C}$ for 3 hours. The obtained solution was stirred for 17 hours at room temperature. To prepare PVAc solutions, $32 \% \mathrm{w} / \mathrm{v}$ of PVAc granules was dissolved in 50\% acetic acid solution and stirred at room temperature for at least 20 hours. PVAc granules were added to the cooled solution of PVA and stirred for 17 hours to prepare the blend solutions of 50:50 by weight $(8.5 \% \mathrm{w} / \mathrm{v})$. CipHCl powder $(5$ or $10 \% \mathrm{w} / \mathrm{w}$ based on the polymer used) was added to the polymer solutions 2 hours before electrospinning.

The electrospinning solution was transferred to a $1 \mathrm{~mL}$ syringe pump with a right angle-shaped needle (gauge 22) attached to it. The resulting fibers were collected on a grounded aluminum plate. All electrospinning processes were carried out at $25^{\circ} \mathrm{C}$. The solution flow rate was 0.8 to $1.3 \mathrm{~mL} /$ hour, the range of applied positive voltage was 15 to $19 \mathrm{kV}$, and the distance between the needle tip and the target was $22 \mathrm{~cm}$. The mat thickness was measured using a thickness tester (Model No 674, range 0-10.00 mm; Hans Baer, Zurich, Switzerland). The thickness of the electrospun nanofiber mats ranged from 300 to $360 \mu \mathrm{m}$. The electrospun nanofiber mats of PVA:PVAc (50:50 by weight) are hereafter termed blend nanofiber mats.

\section{Characterization of electrospun solutions}

Before the electrospinning, the conductivity and viscosity of solutions were measured using a conductivity meter (3540; Jenway, Germany) and a Brookfield digital viscometer
(LVDV-II + PRG, Middleboro, MA), respectively. The average value of three determinations was obtained and reported as mean $\pm \mathrm{SD}$.

\section{Morphology studies}

The morphology of electrospun nanofibers was studied by a scanning electron microscope (XL-30, SEM; Philips, Amsterdam, the Netherlands) after gold coating. The average diameter was determined from the SEM images of each sample. From each image, at least 100 different fiber segments were randomly selected and their diameters were measured using Motic software (v 2, Richmond, BC).

\section{X-ray diffraction studies}

$\mathrm{X}$-ray diffraction (XRD) was used to investigate the effect of electrospinning on the degree of crystallization of the drug as well as the influence of the drug on the crystalline structure of polymer nanofibers. The XRD patterns of pure $\mathrm{CipHCl}$ and blend nanofiber mats with and without drug were determined using a diffractometer ( $\mathrm{X}$ pern; Philips, Amsterdam, the Netherlands). The scanning range varied from $2 \theta=5^{\circ}$ to $40^{\circ}$.

\section{Degree of swelling and weight loss}

The degree of swelling and weight loss of nanofiber mats were calculated by Equations 1 and 2, respectively. Both tests were carried out in the release medium (phosphate buffered saline [PBS], $\mathrm{pH} 7.4$ ) at $37^{\circ} \mathrm{C}$ for 1,4 , and 24 hours.

$$
\begin{gathered}
\text { Degree of swelling }(\%)=\frac{M-M_{d}}{M_{d}} \times 100 \\
\text { Weight loss }(\%)=\frac{M_{i}-M_{d}}{M_{i}} \times 100
\end{gathered}
$$

where $M$ is the weight of swollen nanofibers sample which is wiped dry with filter paper, $M_{d}$ is the dried mass of immersed sample in buffer medium, measured by drying the swollen nanofiber mats in an oven at $40^{\circ} \mathrm{C}$ until constant weight was reached, and $M_{i}$ is the initial dry mass of sample.

\section{In vitro drug release studies}

Drug release from electrospun nanofiber mats was measured by placing a known mass and approximate dimensions $(2.5 \times 2.5 \mathrm{~cm})$ of material into $20 \mathrm{~mL}$ of PBS $(\mathrm{pH} 7.4)$ under constant stirring at $37^{\circ} \mathrm{C}$. At certain time points, a nanofiber sample was taken out of the buffer and placed into a fresh buffer solution. The amount of drug released was determined 
using a UV spectrophotometer at $\lambda_{\max }=271 \mathrm{~nm}$. The release experiments of each sample were performed in triplicate, and average values are reported.

\section{Analysis of drug-release kinetics and modeling}

The release kinetics of drugs from a polymer carrier can be described using the Peppas equation:

$$
M_{t} / M_{\infty}=K t^{n}
$$

where $M_{t}$ is the accumulative amount of drug released at time $t, M_{\infty}$ is the initial drug loading, $K$ is a constant characteristic of the drug-polymer system, and $n$ is the diffusion exponent suggesting the nature of the release mechanism. ${ }^{20}$ In addition to Fickian theory, four more models were used to further analyze the profile of drug release including zero order, first order, Higuchi, and Hixson-Crowell models. ${ }^{40}$

\section{Statistical analysis}

Statistical analysis of data was performed using SPSS software package (v 11; SPSS, Inc, Chicago, IL) by one-way analysis of variance (ANOVA), assuming a confidence level of $95 \%(P<0.05)$ for statistical significance. All data were presented as mean \pm standard deviation.

\section{Results and discussion Morphological structure studies}

During electrospinning, as the liquid jet (polymeric solution) is continuously elongated, the solvent of polymeric

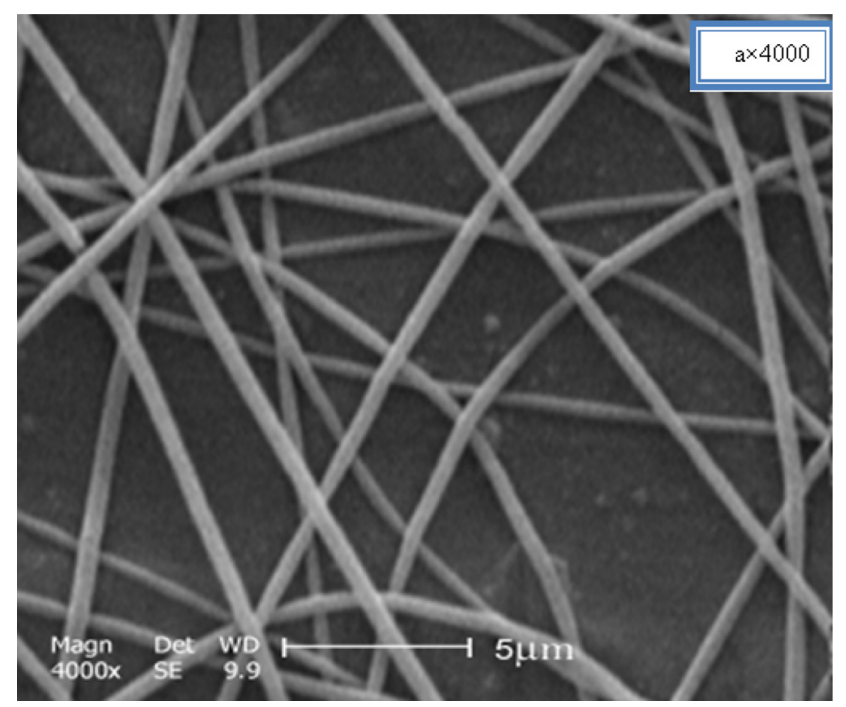

jet is evaporated quickly, phase separation occurs, the jet solidifies, and nanofibers are formed. In this process, nanofibers without solvent are deposited on the collector; however, to ensure that acetic acid did not remain in the nanofiber mats, the mats were dried in a vacuum dryer for 48 hours (at room temperature) to remove the solvent. This method is also reported in other references ${ }^{41-43}$ for removing DMF, trifluoroacetic acid, and 50\% acetic acid from nanofibers. For in vivo experiments, however, some methods such as gas chromatography may be used to verify if solvent remains in the webs after vacuum drying.

As seen in Figure 1, no beaded nanofibers and no drug crystals were detected by electron microscopy on the surface or outside the fibers loaded with the drug, showing that $\mathrm{CipHCl}$ was loaded in the nanofibers, and demonstrating a good compatibility of drug-polymers-solvent ${ }^{19}$ Furthermore, Figures $1 \mathrm{G}$ and $1 \mathrm{H}$ indicate that loading $\mathrm{CipHCl}(10 \% \mathrm{wt})$ into the blend nanofibers (50:50 PVA:PVAc) did not influence the appearance of the nanofiber mats.

The resulting nanofibers also showed that the incorporation of the drug into the nanofibers not only dramatically decreased their average diameter but also reduced the diameter distribution of electrospun nanofibers from 198, 218, and $448 \mathrm{~nm}$ to 145, 180, and $405 \mathrm{~nm}$ for PVA, PVAc, and a blend of PVA/PVAc, respectively, as shown in Figures 1 and 2A. The measurements of viscosity and conductivity of solutions showed that the addition of $\mathrm{CipHCl}$ can significantly decrease solution viscosity (Figure 2B); however, it did not affect solution conductivity (data not shown). Solution viscosity influences the morphological structure and average size of resulting fibers. ${ }^{13,16,20}$

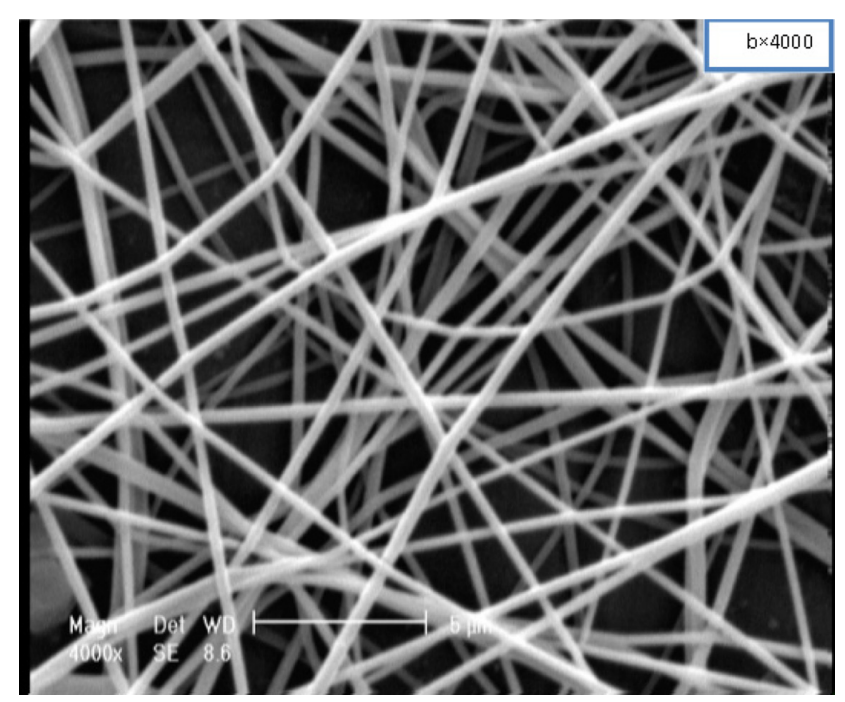

Figure I (Continued) 
(Continued)
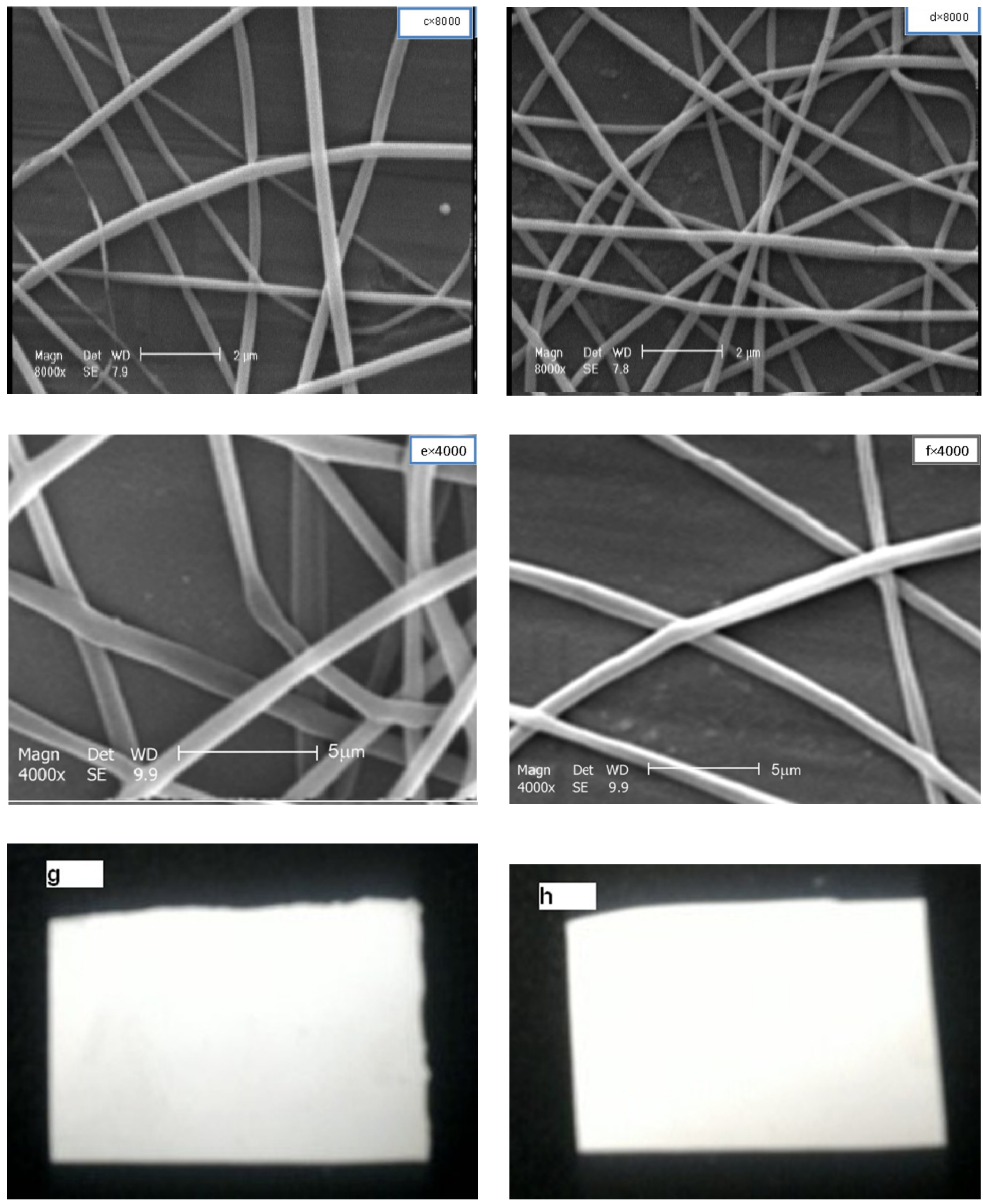

Figure I SEM photographs of electrospun nanofibers without drug and with 10\% w/w drug loaded: A, B) PVA; C, D) PVAc; E, F) a 50:50 blend of PVA/PVAc; G, H) effect of drug loading on appearance of PVA/PVAc blend nanofiber mats.

Abbreviations: $\mathrm{CipHCl}$, ciprofloxacin $\mathrm{HCl}$; PVA, polyvinyl alcohol; PVA/PVAc, polyvinyl acetate. 

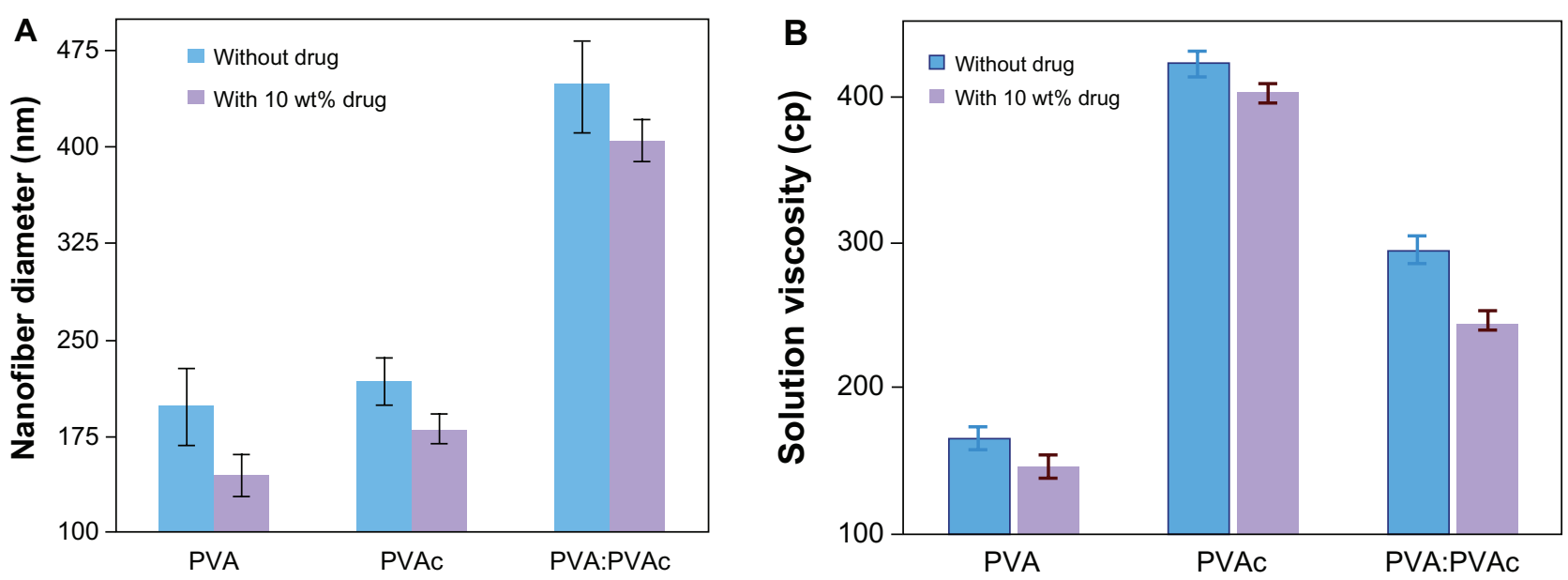

Figure 2 Effect of $\mathrm{CipHCl}$ on $\mathbf{A})$ the diameter of nanofibers $(n=100)$ and $\mathbf{B})$ the solution viscosity $(n=3)$. Abbreviations: PVA, polyvinyl alcohol; PVA/PVAc, polyvinyl acetate.

A possible explanation for the decreasing viscosity of the polymeric solution by addition of $\mathrm{CipHCl}$ is that the drug could be trapped between polymeric chains, and thus acts as a plasticizer. ${ }^{16}$

In general, the electrospun polymeric solution with higher viscosity causes more resistance to elongation during electrospinning. In other words, a decrease in solution viscosity causes the spinning jet to be stretched and elongated more easily. Consequently, finer fibers can result. ${ }^{13}$

\section{XRD studies}

CipHCl powder, pure electrospun PVA:PVAc nanofibers, and $10 \% \mathrm{w} / \mathrm{w}$ drug-loaded PVA:PVAc nanofibers were studied by XRD in order to further illustrate the physical structure and distribution of the drug in the nanofibers. As seen in Figure 3A, CipHCl has several characteristic peaks at $2 \vartheta=19.04,24.8,26.6$, and 29.4 because of its regular crystallization. ${ }^{44}$ XRD pattern of pure PVA:PVAc nanofibers seen in Figure 3B shows typical crystalline peaks at $2 \vartheta=13.9,16.8,18.4,26.3$, and 28.3. Blend nanofiber mats loaded with $10 \% \mathrm{w} / \mathrm{w} \mathrm{CipHCl}$ have the same peaks at $2 \vartheta=13.8,16.9,18.3,26.2$, and 28.2 (Figure 3C), proving that loading the drug did not change the typical crystallinity of the PVA:PVAc blend nanofibers.

Disappearance of the peaks corresponding to the crystalline $\mathrm{CipHCl}$ in PVA:PVAc nanofibers containing $10 \% \mathrm{w} / \mathrm{w}$ drug verified that the drug was dispersed in the polymeric nanofiber matrix in the amorphous phase.

\section{Degree of swelling and weight loss}

Figures 4A and 4B show the degree of swelling and weight loss of PVA, PVAc, and 50:50 blend nanofiber mats in the release medium (PBS, $\mathrm{pH} 7.4$ ) at $37^{\circ} \mathrm{C}$ for 1,4 , and 24 hours. As can be seen, both the degree of swelling and weight loss, which are two important factors contributing to drug release, were in the order of PVA $>$ the blend of PVA:PVAc (50:50) $>>$ PVAc nanofiber mats owing to their hydrophilic-hydrophobic properties.

Although the degree of swelling of the blend nanofiber mats leveled off in comparison with PVA nanofibers, it was still high enough to be used in wound dressings to absorb excess exudates even from deep wounds with high amounts of exudates, compared with other results reported..$^{1-3,23-25}$ The degree of swelling is 203\%, 231\% and $416 \%$ after $1 \mathrm{~h}, 4 \mathrm{~h}$, and $24 \mathrm{~h}$ submersion of the blend nanofibrous mats, respectively.

As demonstrated in Figure 4C, at the earlier stages, the degree of swelling and the thickness of the PVA:PVAc blend nanofiber mats are inversely related, ie, the thinner the nanofiber mats, the greater the degree of swelling. However, the degree of swelling after 24 hours of submersion was greater in the thicker nanofibers.

As calculated from the slope of the lines of the changes of swelling vs time (Figure 4), the swelling rate for the fibers with a thickness of 100 to $140 \mu \mathrm{m}$ is $7.8 \%$ per hour, for 300 to $360 \mu \mathrm{m} \mathrm{9.2 \%} \mathrm{per} \mathrm{hour,} \mathrm{and} \mathrm{for} 690$ to $780 \mu \mathrm{m} \mathrm{10.9 \%} \mathrm{per}$ hour. Nanofiber mats are composed of a great many nanofiber layers: the thicker the mat, the more the layers, and therefore the more compact the interior layers of nanofibers. Over time, the liquid phase has enough time to penetrate into the interior layers and reaches a balance. On the other hand, the more compact areas in thicker nanofibrous mats result in the entrapment of more aqueous molecules in these spaces. As a result, the degree of swelling increases with increased thickness of nanofibrous mats. 


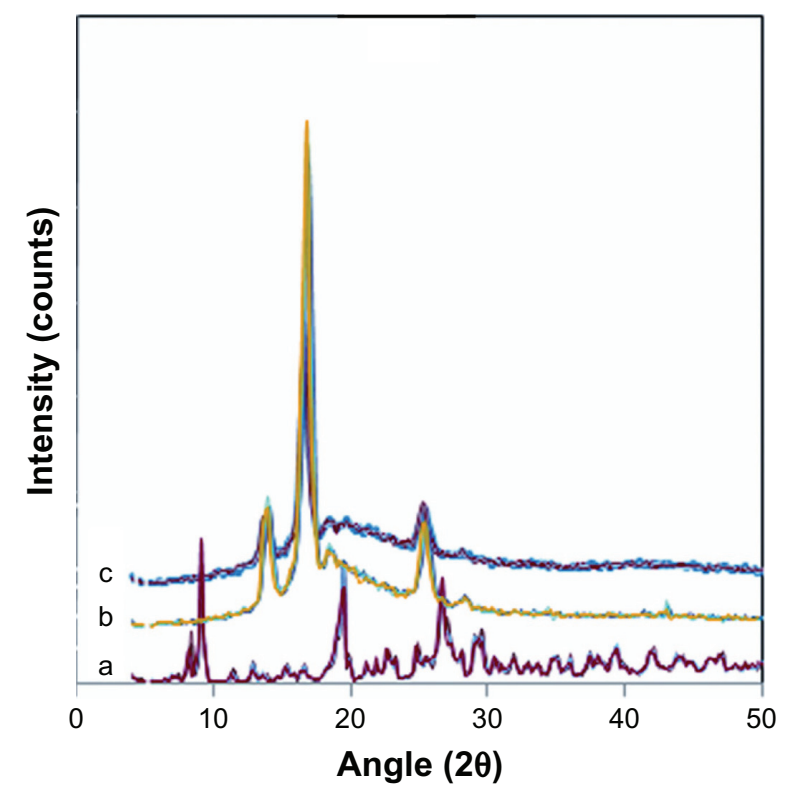

Figure 3 X-ray diffraction of A) $\mathrm{CipHCl}$ powder, B) pure 50:50 PVA/PVAc nanofiber mat, and C) 50:50 PVA/PVAc nanofiber mat loaded with $10 \% \mathrm{w} / \mathrm{w}$ drug. Abbreviations: $\mathrm{CipHCl}$, ciprofloxacin $\mathrm{HCl}$; PVA, polyvinyl alcohol; PVA/PVAc, polyvinyl acetate.

Less aqueous molecules at the earlier stage and more at the later stage can cause a greater increase in the rate of swelling in thicker mats than in thinner mats.

\section{In vitro drug release study from nanofiber mats}

Figure 5 displays the cumulative drug release from PVAc nanofibers loaded with different amounts of $\mathrm{CipHCl}$ up to 1920 hours. The results show a relatively quick initial release. Moreover, increasing the drug content from $5 \%$ to $10 \% \mathrm{w} / \mathrm{w}$ approximately doubled the initial drug release.

We deduced that although aqueous $50 \%$ acetic acid can provide a homogenous solution of PVAc and $\mathrm{CipHCl}$, the incompatibility between hydrophobic PVAc and hydrophilic $\mathrm{CipHCl}^{45}$ would increase the migration of the drug molecules toward the nanofibers surface during the rapid elongation and quick solvent evaporation during electrospinning. These superficial drug molecules can easily diffuse into the aqueous medium and cause an initial quick burst release. As a result, by increasing the drug content, a greater portion of the drug will be located near the surface; therefore, initial release of the drug will be faster. ${ }^{16}$

After a quick burst release, the profiles of release followed a linear fashion with a very slow rate, ie, about $50 \%$ of the drug was released over 80 days of the experiment. This prolonged period of drug release has never been seen in nanofibers before.
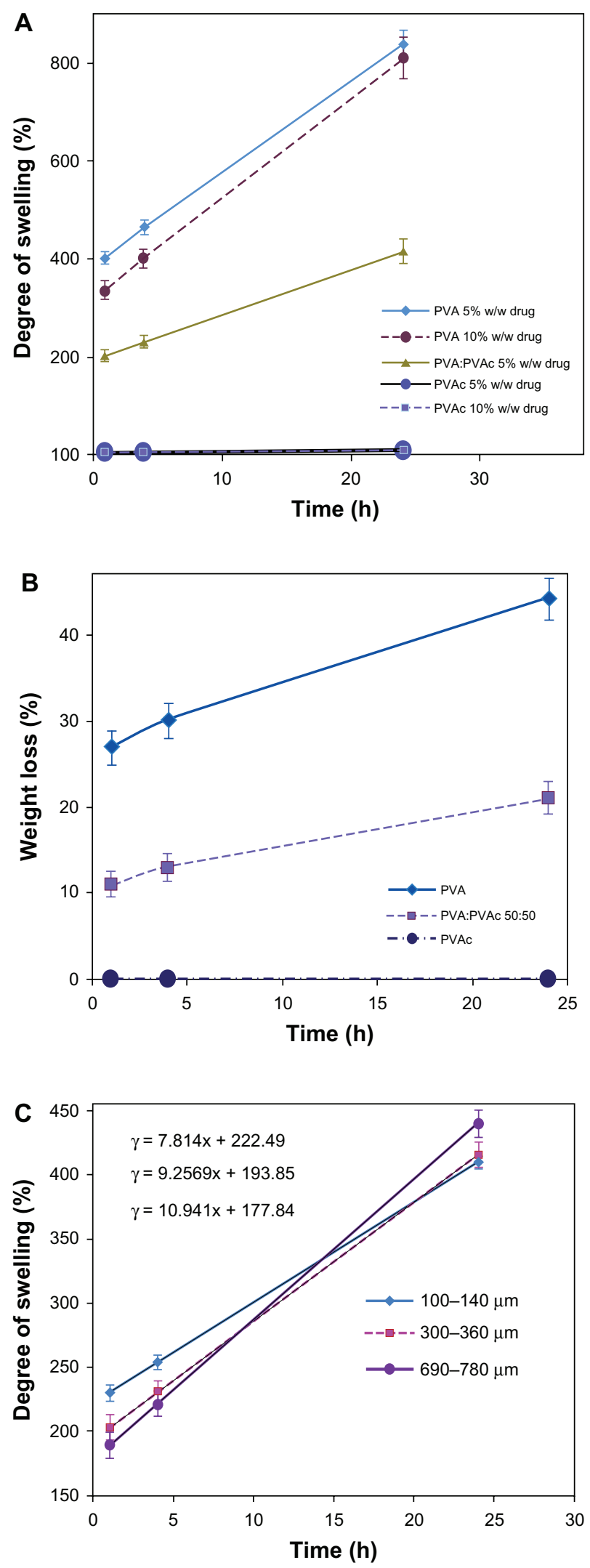

Figure 4 Effect of the type of polymer and/or the drug content A) on the degree of swelling (\%) and B) on the weight loss (\%) of PVA, PVAc and a 50:50 blend nanofiber mat, and $\mathbf{C}$ ) effect of the thickness of blend nanofiber mats on the degree of swelling $(\%)(n=3)$.

Abbreviations: PVA, polyvinyl alcohol; PVA/PVAc, polyvinyl acetate. 


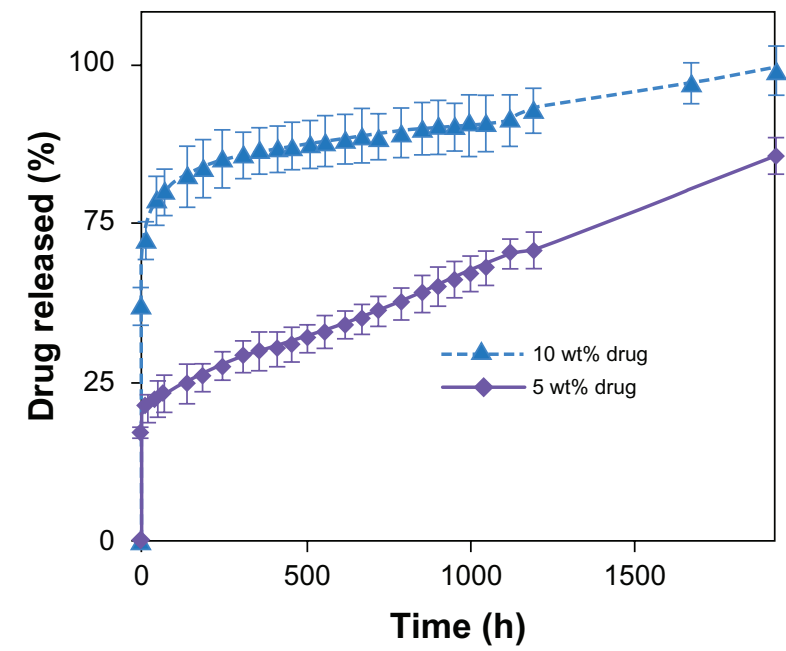

Figure 5 Effect of drug content on release profiles of $\mathrm{CipHCl}$ from PVAc $(32 \% \mathrm{w} / \mathrm{v})$ electrospun nanofiber mats vs time $(n=3)$.

Abbreviations: $\mathrm{CipHCl}$, ciprofloxacin $\mathrm{HCl}$; PVAc, polyvinyl acetate.

The release profiles of $\mathrm{CipHCl}$ from the PVA electrospun nanofibers shown in Figure 6 indicate a high initial release as well as a rapid release rate in which total release time was about 80 hours for both amounts of the drug loaded. It has been found that one of the significant factors contributing to drug release in controlled release systems is the behavior of the matrix loaded with the drug in the release medium. As soon as a PVA matrix is exposed to a liquid medium, it begins to swell (Figure 4A), its molecular chains are solvated, and weight loss occurs (Figure 4B). As a result, the high degree of swelling along with the dissolution of the PVA nanofiber mats, which is attributed to its poor stability in aqueous mediums, causes a quick burst release, as reported previously. ${ }^{8,20,18}$

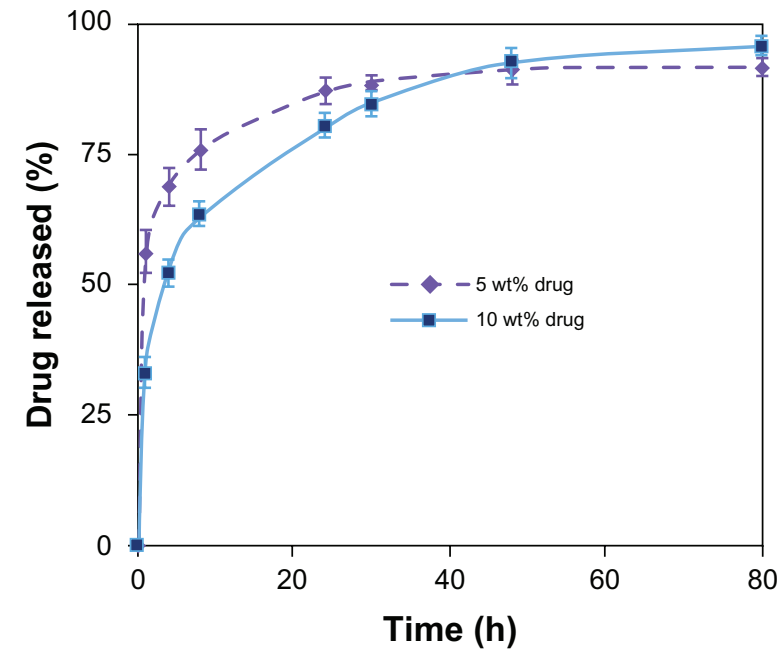

Figure 6 Effect of drug content on release profiles of $\mathrm{CipHCl}$ from PVA ( $5 \% \mathrm{w} / \mathrm{v})$ electrospun nanofiber mats vs time $(n=3)$.

Abbreviations: $\mathrm{CipHCl}$, ciprofloxacin $\mathrm{HCl}$; PVAc, polyvinyl acetate.
According to these profiles, increasing the drug content in PVA nanofiber mats from $5 \%$ to $10 \% \mathrm{w} / \mathrm{w}$ lowered initial drug release and improved the control of the release profile. According to Figure 4A, at the initial experiment stages, a higher drug content $(10 \% \mathrm{w} / \mathrm{w})$ in PVA nanofiber mats results in a lower degree of swelling. Consequently, the penetration of the receiving liquid phase through PVA nanofibers decreases and diffusion of the drug from the nanofibers will be retarded. Kumbar and Aminabhavi ${ }^{46}$ and Sanli et al ${ }^{22}$ studied indomethacine release from polyacrylamide grafted chitosan microspheres and diclofenac sodium release from PVA-sodium alginate beads, respectively. They also observed that increasing the drug content slowed drug release.

Figure 7 compares the effect of polymeric matrix on the initial drug release and the rate of drug release. While PVAc nanofiber mats showed a very slow drug release (regardless of the quick initial release), and PVA nanofiber mats exhibited a rapid rate of drug release and blend nanofiber mats of PVA:PVAc (50:50) a moderate $\mathrm{CipHCl}$ release rate (Figure 7). In other words, incorporation of hydrophobic PVAc into PVA nanofibers decreased the diffusion of release medium into nanofiber mats (Figure 4A) and reduced the weight loss (Figure 4B). As a result, it could be possible to control the rate and the burst release of the drug, in comparison with PVA nanofiber mats, to prolong drug release to at least 250 hours.

Figure 8 illustrates the effect of the thickness of blend nanofiber mats on the release profile if the diameters of the nanofibers were the same. Both the initial release and the rate of drug release from the blend nanofiber mats of

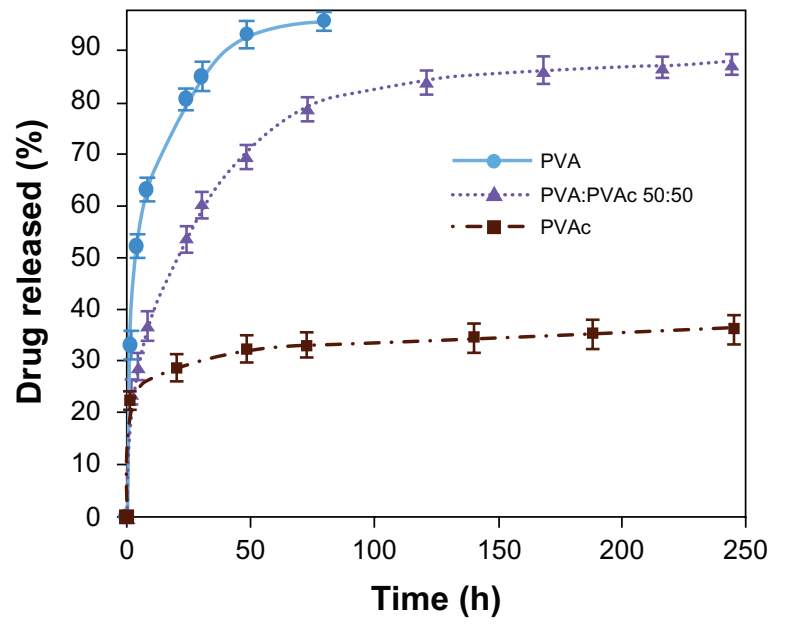

Figure 7 Effect of polymers blending on the release profiles of $\mathrm{CipHCl}$ from medicated electrospun nanofiber mats containing 10\% w/w CipHCl vs time $(n=3)$. Abbreviations: $\mathrm{CipHCl}$, ciprofloxacin $\mathrm{HCl}$; PVA, polyvinyl alcohol; PVA/PVAc, polyvinyl acetate. 


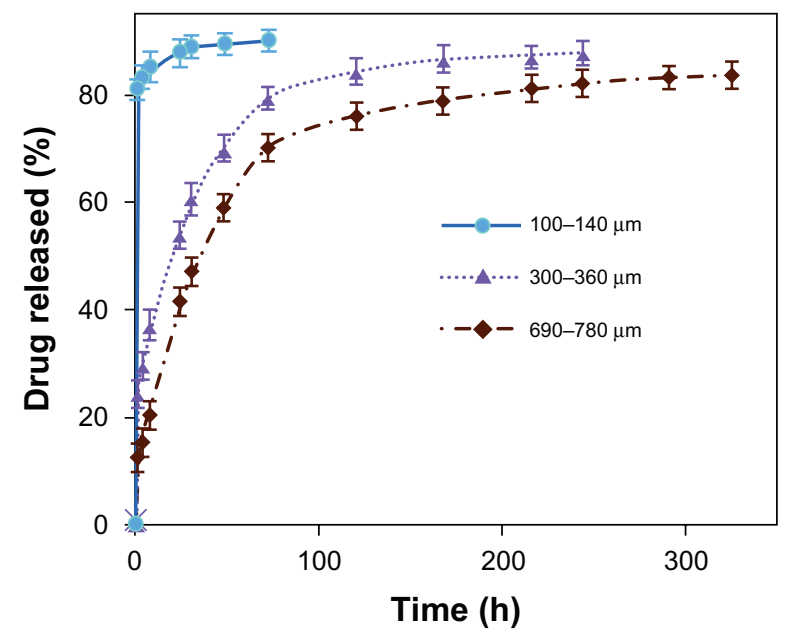

Figure 8 Effect of thickness on the release behavior from medicated electrospun nanofiber mats of PVA:PVAc (50:50) containing 10\% w/w CipHCl vs time $(n=3)$. Abbreviations: $\mathrm{CipHCl}$, ciprofloxacin $\mathrm{HCl}$; PVA, polyvinyl alcohol; PVA/PVAc, polyvinyl acetate.

PVA:PVAc (50:50) significantly decreased as the thickness of the mats increased, which was related to the swelling behavior of these mats (Figure 4C). Furthermore, the total release time was considerably increased, from 72 hours to 325 hours, by increasing the thickness of the mats from 100 to $140 \mu \mathrm{m}$ to 680 to $760 \mu \mathrm{m}$, respectively. A possible explanation lies in the fact that increasing the thickness of nanofiber mats makes the diffusion passage more compact for the drug to be released out of the matrix, which reduces the amount of drug release.

Based on physical evaluations by touch after submersion, PVAc nanofiber mats were too hard to be applied as a wound dressing whereas PVA nanofiber mats and samples of PVA:PVAc (50:50) blend nanofiber mats showed very good flexibility, and could be bent to cover a wound surface. However, for antibiotic delivery systems, a high initial release is necessary to eliminate bacteria before they begin to proliferate. ${ }^{47}$ Also, a sustained release of antibiotic for about 1 week is needed for infected wounds to prevent further proliferation of bacteria. ${ }^{5}$ Blend nanofiber mats of PVA:PVAc (50:50) loaded with $\mathrm{CipHCl}$ with a thickness of more than 300 to $360 \mu \mathrm{m}$ showed a relatively quick initial release as well as a sustained release for at least 10 days; moreover, these mats were able to swell substantially and were flexible and were able to conform to the wound, both of which are requirements for wound dressings.

\section{Analysis of drug release kinetics}

In order to further assess the mechanism of drug release, the cumulative release profiles were analyzed by
Peppas equation, ${ }^{20}$ zero order, first order, Higuchi, and Hixson-Crowell models. ${ }^{40}$ The results of regression analysis of data by the Peppas equation and the values of exponent (n) in that equation are shown in Table 1.

The results indicate the predominance of Fickian diffusion mechanism of release for all formulations, which means that the amount of drug loaded, the type of polymer and the thickness of nanofiber mats did not influence the drug release mechanism.

Generally, drug release from a polymeric matrix is described by a Fickian mechanism when drug diffusion is the main factor in drug release. Diffusion of the drug from a polymeric matrix can occur through two mechanisms: drug diffusion out of the matrix and/or drug diffusion because of matrix degradation. ${ }^{48}$

The regression coefficient analysis of release data for different kinetic models can be seen in Table 2. This table shows that in PVAc nanofibers, the high level of initial release (up to 72 hours) is probably related to the diffusion of the drug located near the nanofibers surfaces, which can be fitted to a Higuchi model. Afterwards, from 72 to 1920 hours, drug release from PVAc nanofibers can be fitted to a Hixson-Crowell model, ie, after 72 hours, the drug was released because of the erosion of the PVAc nanofiber mats. In fact the release behavior can be described by bimodel behavior. Therefore, the erosion mechanism implies diffusion of the drug into the medium. Degradation does not mean complete degradation but involves degradation of nanofibers layer by layer over time, which implies erosion.

For Table 2, Higuchi is the best model to fit the drug release mechanism from PVA and the blend PVA:PVAc (50:50) nanofiber mats, because drug diffusion out of these nanofiber mats plays the main role in drug release.

Table I Diffusion constant $(n)$ and regression coefficient of ciprofloxacin $\mathrm{HCl}$ release from PVA, PVAc, and blend nanofiber mats calculated by Peppas equation $\left(M_{t} / M_{\infty}=k t^{n}\right)(n=3)$

\begin{tabular}{llllr}
\hline $\begin{array}{l}\text { Nanofiber } \\
\text { type }\end{array}$ & $\begin{array}{l}\text { Thickness } \\
\text { of mat }(\mu \mathrm{m})\end{array}$ & $\begin{array}{l}\text { CipHCL } \\
(\% \mathrm{w} / \mathrm{w})\end{array}$ & $\boldsymbol{R}^{2} \pm \mathbf{S D}$ & $\mathbf{N} \pm \mathbf{S D}$ \\
\hline PVA & $300-360$ & 5 & $0.99 \pm 0.04$ & $0.15 \pm 0.00$ \\
& & 10 & $0.99 \pm 0.02$ & $0.27 \pm 0.01$ \\
& & 5 & $0.95 \pm 0.02$ & $0.15 \pm 0.00$ \\
PVAc & $300-360$ & 10 & $0.93 \pm 0.01$ & $0.083 \pm 0.00$ \\
& $100-140$ & & $0.85 \pm 0.06$ & $0.010 \pm 0.01$ \\
PVA:PVAc & $300-360$ & 10 & $0.95 \pm 0.04$ & $0.18 \pm 0.00$ \\
(50:50) & $690-780$ & & $0.97 \pm 0.06$ & $0.19 \pm 0.02$ \\
\hline
\end{tabular}

Abbreviations: $\mathrm{CipHCl}$, ciprofloxacin $\mathrm{HCl}$; PVA, polyvinyl alcohol; PVA/PVAc, polyvinyl acetate. 
Table 2 Regression coefficients of different mathematical models fitted to the release of ciprofloxacin $\mathrm{HCl}$ from different nanofibers with the thickness of 300 to $360 \mu \mathrm{m}(\mathrm{n}=3)$

\begin{tabular}{llllll}
\hline Nanofibers type & CipHCL $(\%$ w/w) & Zero order & First order & Higuchi & Hixon-Crowell \\
\hline PVA & 5 & $0.65 \pm 0.05$ & $0.79 \pm 0.04$ & $0.92 \pm 0.06$ & $0.28 \pm 0.05$ \\
& 10 & $0.71 \pm 0.01$ & $0.85 \pm 0.01$ & $0.94 \pm 0.02$ & $0.36 \pm 0.04$ \\
PVAc before 72 h & 5 & $0.35 \pm 0.04$ & $0.41 \pm 0.06$ & $0.68 \pm 0.02$ & $0.29 \pm 0.03$ \\
& 10 & $0.45 \pm 0.06$ & $0.45 \pm 0.03$ & $0.78 \pm 0.03$ & $0.30 \pm 0.02$ \\
PVAc after 72 h & 5 & $0.96 \pm 0.04$ & $0.93 \pm 0.01$ & $0.94 \pm 0.03$ & $0.98 \pm 0.03$ \\
& 10 & $0.98 \pm 0.05$ & $0.92 \pm 0.01$ & $0.95 \pm 0.02$ & $0.99 \pm 0.06$ \\
PVA:PVAc (50:50) & 10 & $0.66 \pm 0.06$ & $0.72 \pm 0.15$ & $0.90 \pm 0.04$ & $0.38 \pm 0.09$ \\
\hline
\end{tabular}

Abbreviations: $\mathrm{CipHCl}$, ciprofloxacin $\mathrm{HCl}$; PVA, polyvinyl alcohol; PVA/PVAc, polyvinyl acetate.

\section{Conclusion}

Continuous uniform nanofibers of PVA, PVAc, and a blend of these polymers loaded with $\mathrm{CipHCl}$ were successfully electrospun. Addition of the drug reduced the size and narrowed the distribution of electrospun nanofiber diameters, which could be attributed to the decrease in solution viscosity. The amount of drug loaded in nanofibers affected the release behavior and the initial drug release from PVA and PVAc nanofibers. Regardless of the initial rapid release, rate of drug release from PVAc electrospun nanofibrous mats was very slow: about $50 \%$ of the drug was released during 80 days in a linear fashion, whereas the PVA nanofiber mats released the drug within 3 days. Using the blend nanofiber mats of PVA:PVAc (50:50) greatly affected drug release behavior; it reduced the amount of the drug released at earlier stages, and sustained the drug release profile for longer times compared with PVA nanofiber mats. However, blending PVAc with PVA solution made blend nanofiber mats more flexible and more comfortable for use as a wound dressing because of a very significant increase in the degree of swelling comparison with PVAc. Increasing the thickness of the blend nanofiber mats controlled both the initial release and the rate of drug release, and increased the degree of swelling, making the mats suitable for healing deep wounds. All formulations showed Fickian drug release kinetics.

\section{Acknowledgments}

The authors express their sincere gratitude to the Isfahan University of Technology for the financial support. This project was also supported by the Isfahan Pharmaceutical Sciences Research Center (Research Grant No: 285360).

\section{Disclosure}

The authors declare no conflicts of interest in this work.

\section{References}

1. Pal K, Banthia AK, Majumdar DK. Polyvinyl alcohol-glycine composite membranes: preparation, characterization, drug release and cytocompatibility studies. Biomed Mater. 2006;1:49-55.

2. Yu H, Xu H, Chen X, Hao J, Jing X. Medicated wound dressing on poly (vinyl alcohol)/poly(N-vinyl pyrrolidone)/chitosan hydrogels. J Appl Polym Sci. 2006;101:2453-2463.

3. Tsou TL, Tang ST, Huang YC, Wu JR, Young JJ, Wang HJ. Poly (2-hydroxyethyl methacrylate) wound dressing containing ciprofloxacin and its drug release studies. J Mater Sci Mater Med. 2005;16:95-100.

4. Suzuki K, Tanihara M, Nishimura Y, Suzuki K, Kakimaru Y, Shimizu Y. A new drug delivery system with controlled release of antibiotic only in the presence of infection. J Biomed Mater Res. 1998;42:112-116.

5. Boateng JS, Matthews K, Stevens HNE, Eccleston GM. Wound healing dressings and drug delivery systems: a review. J Pharm Sci. 2008;97: 2892-2923.

6. Kang YO, Yoon IS, Lee So, et al. Chitosan-coated poly(vinyl alcohol) nanofibers for wound dressings. J Biomed Mater Res Part B. 2010;92: 568-576.

7. Sreenivasan K. On the restriction of the release of water-soluble component from polyvinyl alcohol film by blending $\beta$-cyclodexterin. $J$ Appl Polym Sci. 1997;65:1829-1831.

8. Öztürk E, Agalar C, Keçeci K, Denkbas EB. Preparation and characterization of ciprofloxacin-loaded alginate/chitosan sponge as wound dressing material. J Appl Polym Sci. 2006;101:1602-1609.

9. Loke WK, Lau SK, Yong LL, Khor E, Sum CK. Wound dressing with sustained anti-microbial capability. J Biomed Mater Res. 2000;53:8-17.

10. Jun J, Yuan DY, Shao WH, Shao ZF, Zhong WY. Preparation and characterization of antibacterial silver-containing nanofibers for wound dressing applications. J US-China Med Sci. 2007;4:52-54.

11. Zhang Y, Lim CT, Ramakrishna S, Huang ZM. Recent development of polymer nanofibers for biomedical and biotechnological applications. J Mater Sci Mater Med. 2005;16:933-946.

12. Khil MS, Cha DI, Kim HY, Kim IS, Bhattarai N. Electrospun nanofibrous polyurethane membrane as wound dressing. J Biomed Mater Res. 2005;67:675-679.

13. Thompson CJ, Chase GG, Yarin AL, Reneker DH. Effects of parameters on nanofiber diameter determined from electrospinning model. Polymer. 2007;48:6913-6922.

14. Huang ZM, He CL, Yang A, et al. Encapsulating drugs in biodegradable ultrafine fibers through co-axial electrospinning. J Biomed Mater Res. 2006;77:169-179

15. Verreck G, Chun I, Rosenblatt J, et al. Incorporation of drugs in an amorphous state into electrospun nanofibers composed of a water-insoluble, nonbiodegradable polymer. $J$ Control Rel. 2003;92:349-360.

16. Zamani M, Morshed M, Varshosaz J, Jannesari M. Controlled release of metronidazole benzoate from poly $\varepsilon$-caprolactone electrospun nanofibers for periodontal diseases. Eur J Pharm Biopharm. 2010;75:179-185.

17. Xu X, Yang L, Xu X, et al. Ultrafine medicated fibers electrospun from W/O emulsions. J Control Rel. 2005;108:33-42. 
18. Kenawy ER, Hay EA, Newehy MHE, Wnek GE. Controlled release of ketoprofen from electrospun poly(vinyl alcohol) nanofibers. Mater Sci Eng A. 2007;459:390-396.

19. Zeng J, Yang L, Liang Q, et al. Influence of the drug compatibility with polymer solution on the release kinetics of electrospun fiber formulation. J Control Rel. 2005;105:43-51.

20. Taepaiboon P, Rungsardthong U, Supaphol P. Drug-loaded electrospun mats of poly(vinyl alcohol) fibers and their release characteristics of four model drugs. Nanothechnol. 2006;17:2317-2329.

21. Kim K, Luu YK, Chang C, et al. Incorporation and controlled release of a hydrophilic antibiotic using poly(lactide-co-glycolide)-based electrospun nanofibrous scaffolds. J Control Rel. 2004;98:47-56.

22. Sanli O, Ay N, Işiklan N. Release characteristics of diclofenac sodium from poly(vinyl alcohol)/sodium alginate and poly(vinyl alcohol)grafted-poly(acrylamide)/sodium alginate blend beads. Eur J Pharm Biopharm. 2007;65:204-214.

23. Seabra AB, Oliveira MG. Poly(vinyl alcohol) and poly(vinyl pyrrolidone) blended films for local nitric oxide release. Biomaterials. 2004;25: 3773-3782.

24. Kim JO, Park JK, Kim JH, et al. Development of polyvinyl alcoholsodium alginate gel-matrix-based wound dressing system containing nitrofurazone. Int J Pharm. 2008;359:79-86.

25. Singh B, Pal L. Development of sterculia gum based wound dressings for use in drug delivery. Eur Polym J. 2008;44:3222-3230.

26. Silvalingam G, Chattopadhyay S, Madras G. Enzymatic degradation of poly( $\varepsilon$-caprolactone), poly(vinyl acetate) and their blends by lipases. Chem Eng Sci. 2003;58:2911-2919.

27. Novoa GAG, Heinämäki J, Mirza S, et al. Physical solid-state properties and dissolution of sustained-release matrices of polyvinyl acetate. Eur J Pharm Biopharm. 2005;59:343-350.

28. Sung JH, Hwang MR, Kim JO, et al. Gel characterization and in vivo evaluation of minocycline-loaded wound dressing with enhanced wound healing using polyvinyl alcohol and chitosan. Int J Pharm. 2010;392:232-240.

29. Don TM, King CF, Chiu WY, Peng CA. Preparation and characterization of chitosan-g-poly(vinyl alcohol)/poly(vinyl alcohol) blends used for the evaluation of blood-contacting compatibility. Carbohyd Polym. 2006;63:331-339.

30. Bonakdar S, Orang F, Rafienia M, Imani R. Comparison of the effect of hydrophilicity on biocompatibility and platelet adhesion of two different kinds of biomaterials. Iranian J Pharm Sci. 2008;4:37-44.

31. Ikada Y, Iwata H, Horii F, et al. Blood compatibility of hydrophilic polymers. J Biomed Mater Res. 1981:697-718.

32. Park KR, Nho YC. Preparation and characterization by radiation of hydrogels of PVA and PVP containing aloe vera. J Appl Polym Sci. 2004;91:1612-1618.

33. Andersen FA. Amended final safety assessment of polyvinyl acetate. J Am Coll Toxicol. 1996;15:166-176.
34. Sadato A, Taki W, Ikada Y, et al. Experimental study and clinical use of poly(vinyl acetate) emulsion as liquid embolisation material. Neuroradiol. 1994;36:634-641.

35. Evans S, Greff RJ, Wright JI. Compositions for use in embolizing blood vessels: US Patent 6342202

36. Dennis CL, Klein GJ, Gebhardt U, Anderson KM, Zillmer GC. Intravascular medical device: US Patent 5607463.

37. Suzawa T, Shirahama H, Fujimoto T. Adsorption of bovine serum albumin onto homo- and copolymer latices. J Colloid Interface Sci. 1982;1:144-150.

38. Beukema RP, Beukema WP, Smit JJ, et al. Efficacy of multi-electrode duty-cycled radiofrequency ablation for pulmonary vein disconnection in patients with paroxysmal and persistent atrial fibrillation. Europace. 2010;12:502-507.

39. Hoshi T, Sawaguchi T, Konno T, Takai M, Ishihara K. Preparation of molecular dispersed polymer blend composed of polyethylene and poly(vinyl acetate) by in situ polymerization of vinyl acetate using supercritical carbon dioxide. Polymer. 2007;48:1573-1580.

40. Costa P, Lobo JMS. Modeling and comparison of dissolution profiles. Eur J Pharm Biopharm. 2001;13:123-133.

41. Wang Q, Dong Z, Du Y, Kennedy F. Controlled release of ciprofloxacin hydrochloride from chitosan/polyethylene glycol blend films. J Carbohyd Polym. 2007;69:336-343.

42. Landau O, Rothschild A, Zussman E. Processing-microstructureproperties correlation of ultrasensitive gas sensors produced by electrospinning. Chem Mater. 2009;21:9-11.

43. Meng Z X, Zheng W, Li L, Zheng Y F. Fabrication, characterization and in vitro drug release behavior of electrospun PLGA/chitosan nanofibrous scaffold. Mater Chem Phys. 2011;125:606-611.

44. Steele J, Neufeld R. Encapsulation of crosslinked gelatin nanofibers to better mimic ECM conditions within alginate microparticles, XVIIth International Conference on Bioencapsulation. Porto, Portugal, October $1-2,2010$.

45. Breda SA, Jimenez-Kairuz AF, Manzo RH, Olivera ME. Solubility behavior and biopharmaceutical classification of novel high-solubility ciprofloxacin and norfloxacin pharmaceutical derivatives. Int J Pharm. 2009;371:106-113.

46. Kumbar SG, Aminabhavi TM. Synthesis and characterization of modified chitosan microspheres: effect of the grafting ratio on the controlled release of nifedipine through microspheres. J Appl Polym Sci. 2003;89:2940-2949.

47. Park SJ, Kim KS. Influence of hydrophobe on the release behavior of vinyl acetate miniemulsion polymerization. Colloids Surf B. 2005; 45:52-56.

48. Rosenberg R, Sevenney W, Seigel S, Dan N. Anomalous release of hydrophilic drugs from poly( $\varepsilon$-caprolactone) matrices. Mol Pharm. 2007;4:943-948.
International Journal of Nanomedicine

\section{Publish your work in this journal}

The International Journal of Nanomedicine is an international, peerreviewed journal focusing on the application of nanotechnology in diagnostics, therapeutics, and drug delivery systems throughou the biomedical field. This journal is indexed on PubMed Central,

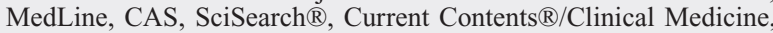

\section{Dovepress}

Journal Citation Reports/Science Edition, EMBase, Scopus and the Elsevier Bibliographic databases. The manuscript management system is completely online and includes a very quick and fair peer-review system, which is all easy to use. Visit http://www.dovepress.com/ testimonials.php to read real quotes from published authors. 